\title{
RESEARCH ON LOW ALTITUDE IMAGE ACQUISITION SYSTEM
}

\author{
Hongxia Cui ${ }^{1,2}$, Zongjian Lin $^{2}$, Jinsong Zhang ${ }^{3, *}$ \\ ${ }^{1}$ Department of Information Science and Engineering, University of Bohai, Jinzhou, Liaoning \\ Province, 121000, China \\ ${ }^{2}$ Chinese Academy of Surveying and Mapping, Beijing, 100039, China \\ ${ }^{3}$ Department of public information network security supervise, Jinzhou police station, Jinzhou, \\ Liaoning Province, 121000, China \\ * Corresponding author, Address: Department of public information network security supervise, \\ Jinzhou police station, Jinzhou, Liaoning Province, 121000, China, Tel: +86-0416-8919456; \\ Email:Inchx316@sohu.com
}

\begin{abstract}
In order to acquire quick, high resolution, affordable aerial photographs, an unmanned helium filled airship system equipped with a non-metric digital camera was developed in Chinese Academy of Surveying and Mapping. To meet the potential demand of large scale photogrammetric applications, the non-metric digital camera was calibrated rigourously based on the bundle adjustment with additional parameters and the workflow for generating digital orthophotos was proposed. At the paper end, the promising results obtained from an application with the system are shown and evaluated.
\end{abstract}

Keywords: UAV (unmanned aerial vehicle), photogrammetry, airship, camera, computer vision

\section{INTRODUCTION}

For the applications of aerial photogrammetry and remote sensing, the lack of appropriate image acquisition methods is usually one of the most important points of interest. Compared with classical flight campaigns, other UAV-based platform such as kite, balloon, airship and remote-controlled model helicopter or unmanned aircraft seems to provide a low-cost solution for quick, affordable image acquisition of area with small size (especially in urban areas). 
During past decades, to meet the increasing demand for low-cost, highresolution and large-scale imagery, UAV-based systems equipped with digital cameras have been used in many fields including 3D building reconstruction (Eisenbeiss et al., 2004), precision agriculture (Eisenbeiss et al., 2004), rapid emergency response operations (Davison et al., 2003), 3D vector map production (Lacroix et al., 2004), etc. Researchers in Chinese Academy of Surveying and Mapping have developed an unmanned aircraft (Cui et al., 2004) and an unmanned airship to provide low altitude platforms for diverse applications.

\section{SYSTEM OVERVIEW}

The UAVF-RS (unmanned aerial vehicle for remote sensing) system developed in Chinese Academy of Surveying and Mapping consists of the remote controlled helium-airship, the photographic system and the ground station.

\subsection{Helium airship}

The unmanned helium-airship (Fig. 1) consists of an envelope, a gondola, two fins, an electrical system.

It is designed to be able to carry photographic system. It is designed to fly in areas near to ground between $20 \mathrm{~m}$ and $1,000 \mathrm{~m}$ flying height. The heliumairship is controlled by the model uav pilot.

Two fins are attached to the envelope. There is the airship's control system with its electrical supply, fuel and propulsion in the gondola. Two petrol engines provide the propulsion. The electrical system is comprised of a radio receiver, a radio transmitter, some actuators, an onborad GPS system and power supply (two sealed lead-acid batteries).

The technical characteristics of the airship in this paper are the following:

Volume: 66cu.mts

Length: 12 meter

Maximum payload weight (Camera): $5 \mathrm{~kg}$

Maximum take-off weight: $72 \mathrm{~kg}$

Range of control: $30 \mathrm{~km}$

Endurance: $2.5 \mathrm{hrs}$

Maximum altitude: $1.0 \mathrm{~km}$

Maximum velocity: $20 \mathrm{~m} / \mathrm{sec}$ 


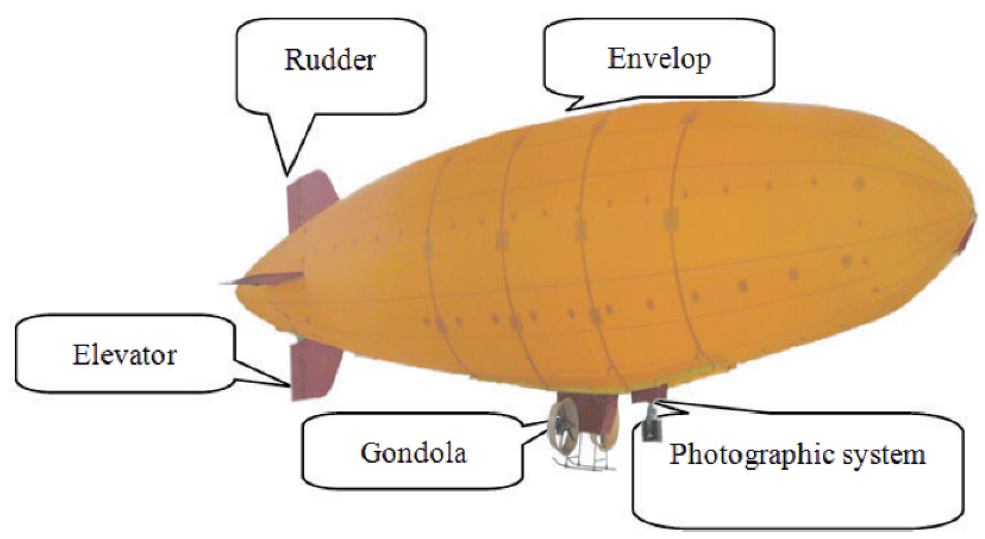

Fig. 1. Unmanned helium-airship

\subsection{Photographic system}

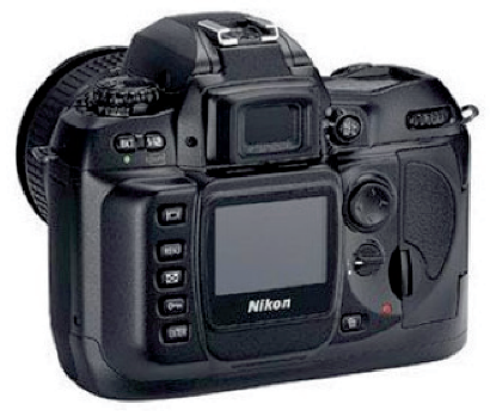

Fig. 2. Nikon D100 camera

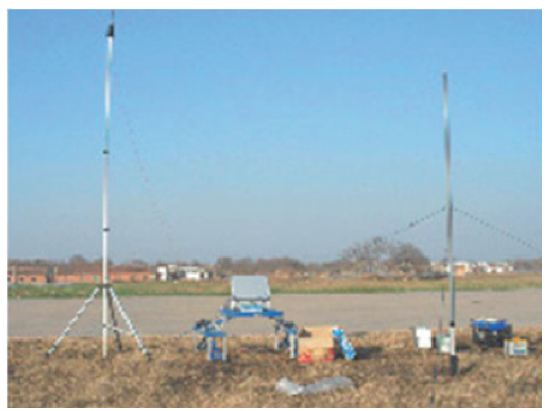

Fig. 3. Ground station overview

The Nikon D100 camera with lens of $20 \mathrm{~mm}$ focal length (Fig. 2) is mounted on a two axis pan \& tilt platform under the airship which is completely self-leveling. An automatic control system is developed to ensure the camera exposuring normally. Since the airship is always moving in the breeze, fast shutter speeds are needed.

A small video camera is placed up to the viewfinder of the Nikon D100 camera and is wired to a video transmitting unit. Therefore the on-board color-micro video camera provides the operator on the ground with an image of the area to be photographed.

\subsection{Ground station}

The Ground station (Fig. 3) includes a video monitor, a video receiver, a data receiver, a flight navigation computer and various switches for 
operating the airship and photographic system onboard. The flight navigation computer receives GPS data from the airship via data receiver unit and forms the flight trace on a real time basis. The pilot compares the flight trace with the flight plan and controls the airship via radio control unit. In addition, the monitor shows exactly what the viewfinder of the photo camera sees.

\section{CAMERA CALIBRATION}

The Nikon D100 camera was calibrated using the bundle adjustment with additional parameters method (Weng et al., 1990). Because of being a nonmetric camera, its distortion calibration accuracy is evaluated in detail.

\subsection{Bundle adjustment with additional parameters}

The Bundle adjustment with additional parameters method is normally divided into four steps: First, Object space coordinates of control points on some calibration bar in a laboratory were measured by use of total station. Second, 8 images of the control points from four different positions were taken by the camera with the lens fixed at infinity focus. Third, coordinates of control points on each image are (semi-) automatically extracted. Fourth, calibration is implemented by use of bundle adjustment method.

According to the two image datasets captured on Aug. $15^{\text {th }}$ and Oct. $11^{\text {th }}$ in 2005 separately, the camera was calibrated two times by the software developed in this paper (Fig. 4).The calibration results are shown in Table 1.

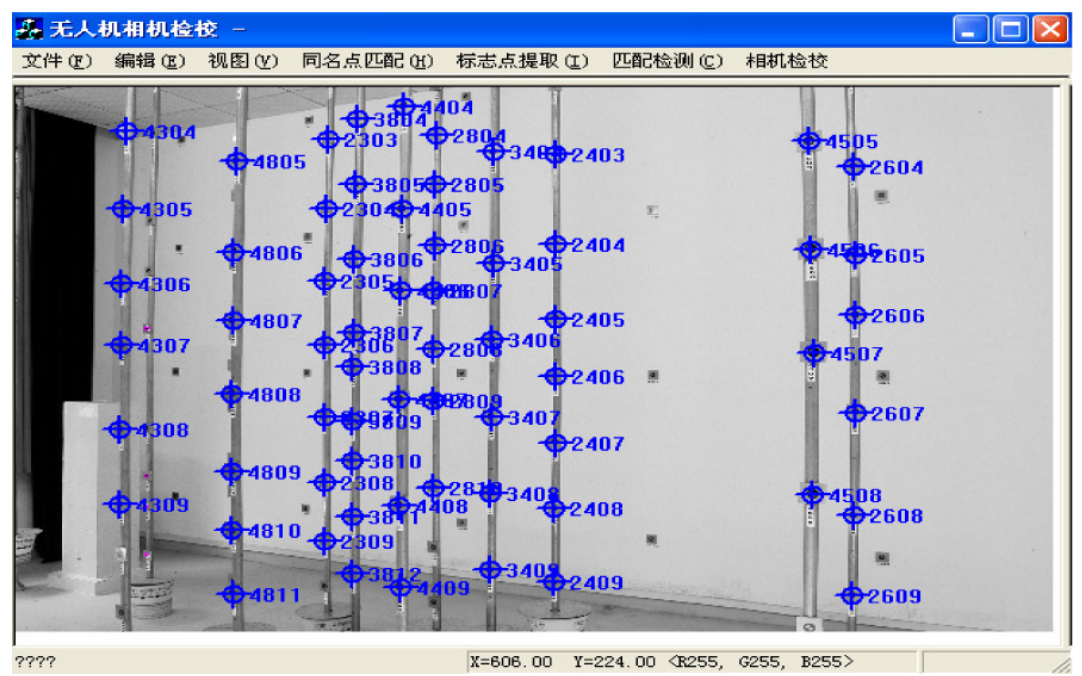

Fig. 4. Camera calibration software 
Table 1. Parameters of interior orientation and distortion

\begin{tabular}{|c|c|c|c|c|}
\hline & \multicolumn{2}{|c|}{ Aug. $15^{\text {th }}, 2005$} & \multicolumn{2}{|c|}{ Oct. $11^{\text {th }}, 2005$} \\
\hline & value & $\square \sigma_{0}$ & value & $\square \sigma_{0}$ \\
\hline$f($ pixel) & 2617.12 & 0.42 & 2617.31 & 0.76 \\
\hline $\mathrm{x} 0$ (pixel) & 1505.27 & 0.21 & 1505.8 & 0.13 \\
\hline y0 (pixel) & 1000.45 & 0.2 & 1000.4 & 0.14 \\
\hline $\mathrm{k} 1\left(\right.$ pixel $\left.^{-2}\right)$ & $1.58 \times 10^{-8}$ & $3.69 \times 10^{-10}$ & $1.60 \times 10^{-8}$ & $1.67 \times 10^{-10}$ \\
\hline $\mathrm{k} 2\left(\right.$ pixel $\left.^{-4}\right)$ & $-1.26 \times 10^{-15}$ & $6.41 \times 10^{-16}$ & $-1.42 \times 10^{-15}$ & $4.29 \times 10^{-16}$ \\
\hline p1 $\left(\right.$ pixel $\left.^{-2}\right)$ & $-3.09 \times 10^{-7}$ & $7.10 \times 10^{-8}$ & $-2.68 \times 10^{-7}$ & $9.08 \times 10^{-9}$ \\
\hline p2( pixel $\left.^{-2}\right)$ & $8.25 \times 10^{-8}$ & $3.02 \times 10^{-8}$ & $8.30 \times 10^{-8}$ & $3.49 \times 10^{-8}$ \\
\hline b1 $\left(\right.$ pixel $\left.^{-1}\right)$ & $4.87 \times 10^{-4}$ & $1.29 \times 10^{-6}$ & $3.64 \times 10^{-4}$ & $3.29 \times 10^{-6}$ \\
\hline b2 $\left(\right.$ pixel $\left.^{-1}\right)$ & $9.88 \times 10^{-5}$ & $4.98 \times 10^{-6}$ & $7.83 \times 10^{-5}$ & $7.01 \times 10^{-6}$ \\
\hline
\end{tabular}

Where the terms of $\mathrm{f}$ represents focal length, $(\mathrm{x} 0, \mathrm{y} 0)$ the location of the principal point, $\mathrm{Ki}$ the coefficients of radial distortion and $\mathrm{r}$ the radial distance. Pi are the coefficients of the decanting distortion. The scale parameter b1 models a no-square pixel size and shear parameter b2 compensates for a nonorthogonality in the pixel array.

\subsection{Distortion evaluation}

During adjustment, the coordinates of check points are deemed unknown. Finally, adjustment results are compared with observation of total station to check accuracy. The relative accuracy is shown in Table 2, it is obvious that although the accuracy in the $\mathrm{Z}$ (height) direction is not as high as that in the planar direction.

Table 2. Relative accuracy

\begin{tabular}{ccc}
\hline & Aug. $15^{\text {th }}, 2005$ & Oct. $11^{\text {th }}, 2005$ \\
\hline Z & $1 / 6230$ & $1 / 6289$ \\
Planar & $1 / 8474$ & $1 / 8264$ \\
\hline
\end{tabular}

There are four principal sources of departure from collinearity, which are "physical" in nature (Fraser et al., 1999). These are the radial lens distortion, de-centering lens distortion, image plane un-flatness, and in-plane image distortion. Radial lens distortion is calculated by polynomial series, equation (1) (Kraus et al., 2000). The term $K_{1}, K_{2}$ are considered in this paper.

$$
\begin{aligned}
& \Delta x_{R D}=\left(x-x_{0}\right) r^{2} k_{1}+\left(x-x_{0}\right) r^{4} k_{2} \\
& \Delta y_{R D}=\left(y-y_{0}\right) r^{2} k_{1}+\left(y-y_{0}\right) r^{4} k_{2}
\end{aligned}
$$

where

$$
r=\sqrt{\left(x-x_{0}\right)^{2}+\left(y-y_{0}\right)^{2}}
$$

Decentering distortion which can be acquired by equation (2) is the second category of lens distortion because of lack of centering of the lens elements along the optical axis. 


$$
\begin{aligned}
& \Delta x_{D D}=\left(2\left(x-x_{0}\right)^{2}+r^{2}\right) p_{1}+2\left(x-x_{0}\right)\left(y-y_{0}\right) p_{2} \\
& \Delta y_{D D}=2\left(x-x_{0}\right)\left(y-y_{0}\right) p_{1}+\left(2\left(y-y_{0}\right)^{2}+r^{2}\right) p_{2}
\end{aligned}
$$

Out-of-plane image deformation is usually ignored. In-plane distortions include distortion caused by differential scaling between $\mathrm{x}$ and $\mathrm{y}$ image coordinates and non-orthogonality between image axes. These distortions are usually denoted "affine deformations" and can be mathematically obtained by equation (3).

$$
\Delta x_{C D}=\left(x-x_{0}\right) b_{1}+\left(y-y_{0}\right) b_{2}
$$

Thus, the total systematic distortion can be described by equation (4).

$$
\begin{aligned}
& \Delta x=\Delta x_{R D}+\Delta x_{D D}+\Delta x_{C D} \\
& \Delta y=\Delta y_{R D}+\Delta y_{D D}+\Delta y_{C D}
\end{aligned}
$$

Conclusions can be drawn by computing different distortion of every image point. First, the total distortion is up to 69.67 pixel and radial distortion is always over $97 \%$. Second, the decentering distortion can not be ignored for the non-metric camera used in the paper. Third, the affine distortion is less than 0.1 pixel which will be not considered during the following image processing.

\section{PHOTOGRAMMETRIC APPLICATION}

With the developed airship and the camera calibrated in this paper, the low altitude images of a test project area have been taken. The flight plan and the flight trace of the airship is illustrated in Fig. 5.

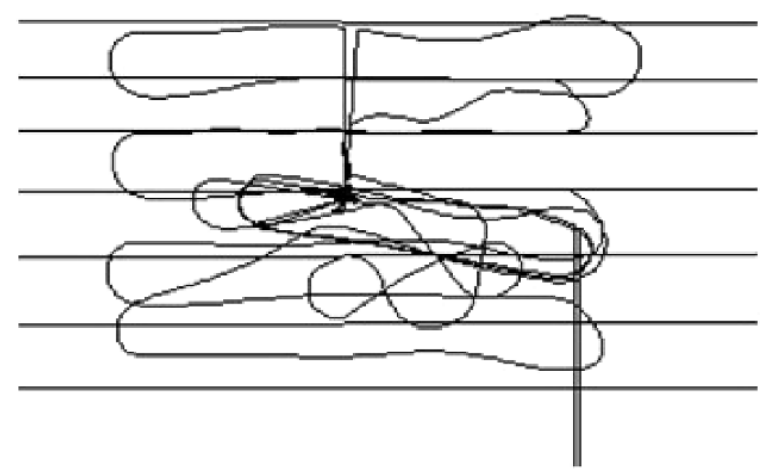

(straight lines: flight plan; curves: flight trace)

Fig. 5. Test flight 
The airship-based system enables to acquire low altitude digital images. To deal with these images for photogrammetric application, the specific software has been developed. The workflow of the software to produce photogrammetric products is aerial triangulation, block adjustment, image matching, DEM production and 3D stereo models (Cramer et al., 2003). Currently, the software enables delivery of seamless high resolution orthophotos and 3D vector maps. Especially, one point on the ground can be imaged up to 15 images because the strips of images have at least $80 \%$ forward overlap and at least $20 \%$ side overlap. Based on such images, we propose to detect and eliminate the blunder and increase the number of multi-ray points robustly by means of relative orientaton, trifocal tensor (Hartley et al., 2003) as well as free net bundle adjustment step by step. Further, over 100 points at 1:500 maps have been measured three dimensionally in CAD-Software. Through comparing the coordinates of these points with those derived from the orthophoto, conclusions can be drawn that the accuracy meets that required for national map accuracy standards at scale of 1:2000. A further control was made by placing the raster map at scale of 1:500 over the orthophoto (Fig. 6).

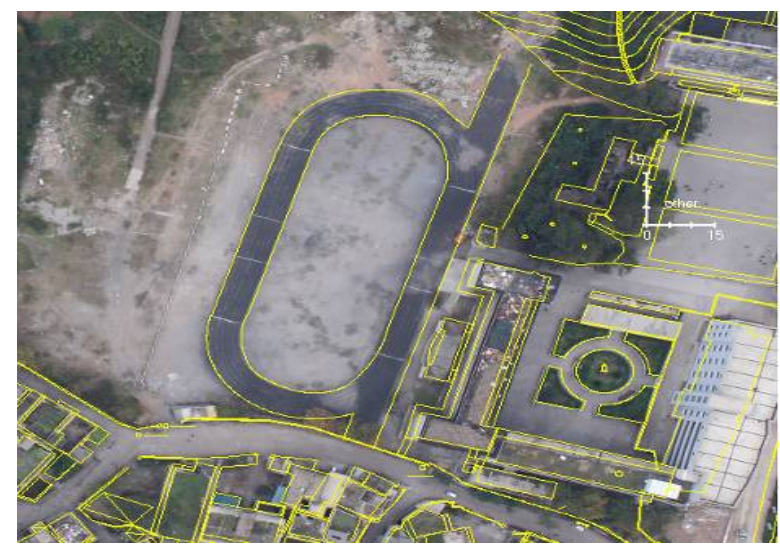

Fig. 6. 1:2000 Orthophoto with 1:500 raster map

\section{CONLUSIONS}

From what has been discussed above, we may safely draw conclusions as follows:

(1) The advantages of this system over traditional aerial photogrammetric system with large format metric cameras are its quick, affordable image acquisition of area with small size (especially in urban areas). 
(2) Non-metric camera is able to be applied in photogrammetric application at large scale after rigorous calibration. However, the decentering distortion can not be ignored and the affine distortion is not considered during image processing.

(3) The high redundancy of digital images is helpful to a fully automated workflow for photogrammetric production.

\section{REFERENCES}

Cramer M. GPS/inertial and digital aerial triangulation - recent test results. Photogrammetric Week , 2003:161-172.

Cui HX, Lin ZJ, Sun J. UAVRS-II unmanned air vehicle remote sensing system. Proceedings of the third international symposium on instrumentation science and technology, 2004, 2:355-359.

Davison AJ. Real-time simultaneous localization and mapping with a single camera. IEEE Int. Conf. on Computer Vision, 2003:1403-1410.

Eisenbeiss H. A mini unmanned aerial vehicle (UAV): system overview and image acquisition. International Workshop on Processing and visualization using high-resolution imagery, 2004.

Fraser C. Digital camera self-calibration. ISPRS Journal of Photogrammetry and Remote Sensing. 1997, 52:149-159.

Hartley R, Zisserman A. Multiple View Geometry in Computer Vision. Cambridge University Press, 2003.

Herwitz SR, Johnson LF, Dunagan SE. Demonstration of UAV-based imaging for agricultural surveillance and decision support. Computers and Electronics in Agriculture, 2004, 44:49-61.

Kraus K. Choice of Additional Parameters. Photogrammetry Advanced Methods and Applications. 2000, 2:133-136.

Lacroix S, Kung IK. High resolution 3D terrain mapping with low altitude imagery. 8th ESA Workshop on Advanced Space Technologies for Robotics and Automation, 2004.

Weng J, Cohen M, Claibration of stereo cameras Using a Non linear distortion model (CCD sensory). Proc. 10th. conf. Pattern recognition, 1990:246-253. 